\title{
Research on the Strategies of Mobile Communication
}

\author{
Yu Wang \\ College of Liberal Arts Changchun University of Science and Technology, China.
}

\begin{abstract}
Keywords: Mobile phone animation communication strategy.
\end{abstract}
\begin{abstract}
This paper starts from the characteristics of mobile phone animation in China at the present stage and systematically analyzes it in theory. In the research process, the traditional animation communication characteristics were used as a control, and the impact of mobile communication methods on animation production methods, audience groups, and operation methods was analyzed. The results showed that mobile phones with strong interaction, speed, and intelligence were used as mobile phones
\end{abstract}

\section{Introduction}

As the largest potential mobile animation market in China, compared with other countries with advanced animation distribution, there are still certain problems in mobile animation, such as poor product originality, insufficient content, insufficient quantity, etc., and China's animation development lacks copyright protection. The establishment of a well-organized commercial mobile phone animation distribution model is an important factor constraining its development.

\section{The Concept of Mobile Animation}

Mobile phone animation refers to the use of mobile phones as a carrier to use the Internet to upload, download, and forward animation products. Users search for their favorite anime content on mobile phones and online platforms and save it on their own mobile phones.

\section{The Existing Problems of Mobile Phone Animation in China}

\subsection{The Originality of Animation Works is Poor}

The majority of mobile animation production in China comes from works that are spontaneously designed by the masses. These cartoon works lack a uniform style, lack good guidance, and have a uniform content, a rough quality, and a low artistic value. Even if the animation works developed by a professional team are difficult to reflect innovation and attraction, they cannot meet the high requirements of the era for the content of the cultural industry[1]. They neither promote the outstanding traditional culture of our country nor show excellent modern culture. In the international animation industry, several works based on traditional Chinese culture originated from foreign production teams, such as "Kung Fu Panda" and "Mulan". In addition, some of the works incorporate Chinese elements in their creations, but in fact they promote their own culture. This type of work has attracted a large number of Chinese audiences and has also had a great influence on the world.

\subsection{Mobile Phone Users Have Limited Acceptance of Animation}

Although the concept of mobile animation has long been introduced to the country and given greater expectation, it is considered a dark horse in the field of mobile value-added services. However, compared with other mobile-related media, the development of the mobile animation industry is relatively backward. Most mobile phone users do not have the habit of watching animations using mobile phones. This is because the service providers have not been able to provide personalized services according to the mobile users' consumer needs[2]..

\subsection{Cultural Industry Property Awareness is Weaker}

Mobile phones are known for their convenient downloading and dissemination capabilities, which is also the reason for the infringement. China's mobile animation industry lacks awareness of property rights and has not completed a system of authentication and encryption. As a result, mobile phone 
users can arbitrarily process, distort, and forward original animation products, causing plagiarism and imitation imaginations to continue to be prohibited. At the same time, there is a lack of laws in the cultural industry, and there is weak supervision. Once property rights disputes arise, it is often difficult to resolve them in a short period of time. This seriously damages the interests of the creator of animation works and the provider of mobile animation content, making the investment enthusiasm and the recognition of creative products greatly reduced, which also hinders the development of mobile animation. 2.4 The defects of mobile phone animation itself

Mobile phone models, performance, screen resolution and other hardware conditions are uneven, and the constraints of these hardware facilities make some users unable to become consumer groups of mobile animation. These people's mobile anime viewing experience is poor, inhibiting their use of mobile phones to comics. The enthusiasm for product consumption will hinder the popularization and development of mobile animation in China in the long run. Most of the screen resolution and brightness of the mobile phone are different. The original content and high-definition picture quality cannot be perfectly displayed on the mobile phone. It also indirectly causes the mobile phone animation supplier to abandon the mobile phone transmission route and find consumers more acceptable. , Can present the original picture quality of animation works

\section{Optimize the Strategy of Mobile Animation in China}

\subsection{Creating with Local Culture}

China's outstanding historical allusions, folk stories, classic classics, etc. can all be exploited and used by creative personnel. The more ethnic the world is, the more world-renowned it is, and from the perspective of several international anime works with national style, most of them have received praise from the audience. However, due to the unsatisfactory development speed of the animation industry in China, many of China's outstanding themes have been quickly achieved by foreign creative teams. For instance, the "Three Kingdoms", a popular folk novel by Chinese people, has been used by Japanese anime companies in the creation of content. The massive influx of Chinese culture into foreign countries has no longer been a new issue in the creation of products. This has sounded an alarm for the development and spread of the animation industry in China [3].

\subsection{Cultivate a Complete Industrial Chain with Animation Brand Awareness}

Innovation is the spirit that we urgently need in this era. It is also recognized as a sunrise industry in the commercial field, and the mobile phone animation distribution is even more desirable. The establishment of the industrial chain has lost its initial and most important impetus. Therefore, a creative animation image and a creative animation brand are the key to ensuring the mobile animation communication and the foundation for the long-term stable development of mobile animation. The classic animation image will be rooted in the viewer's heart. In such a situation, combined with the mobile phone, the audience will be more willing to accept the mobile animation communication, which will also bring rich profits to the development team.

\subsection{Improve the Level of Animation Production}

Mobile phone animation is a culturally-intensive creative industry. It itself embodies both technology and art. In its later development, the original art is all done, and advanced technology is the means of promotion. Any new media industry can not deny the power of technology, mobile animation communication is even more so, in the mobile phone animation, technology supports the creation, reception, impact and other key links. To achieve mobile phone users willing to watch animation works on mobile phones, first of all have a clear picture quality and smooth viewing experience, then require the animation production team to optimize the original technology and develop excellent works of high simulation, high degree of reduction [4 ].

\subsection{Strengthen the Specification of Mobile Animation}

The development of mobile animation depends on the support and wisdom of the group, but it must also be properly guided to make it clear of the consequences and harm of poorly distributed animation works. Only the professional animation creation team and the masses' spontaneous creations have the right direction. Only in this way can China's mobile animation industry develop into benign development. Benign development is the key to the animation of mobile phones. In the 
process of the gradual formation of the animation communication industry chain, the purification of the source of creation determines the future of this industry, so strengthen the opponent's mobile phone. The supervision and filtering of animation communication is the top priority of the industry.

\section{Conclusion}

This article sets out from the realistic environment for the development of mobile animation in China, analyzes the various factors that affect the development of mobile animation, draws on international advanced experience in animation distribution, and provides more targeted analysis of the shortcomings of China's animation distribution. Although China has the world's largest mobile terminal use group, the development of mobile phone animation distribution is not satisfactory compared with several advanced countries in the world. Mobile phone animation distribution is at a disadvantage. In order to achieve the sharing of resources, we will incorporate local cultural factors in animation and enhance the value of animation works. Only in this way can we attract more motivated cartoon users. At the same time, we should learn more about the successful development experience in the process of mobile animation, such as the development of animation in our neighboring countries, South Korea, Japan, or the United States across the ocean. In order to seek long-term sustainable development, China's mobile animation communication industry is even better suited to its own development. If we can fundamentally solve these problems, the development prospects and space for China's mobile animation industry will be immeasurable, relying on a huge number of mobile phone users in China, I believe in the near future, the development of China's mobile animation industry will show a new The situation.

\section{Acknowledgements}

The author would like to give her thanks to Jilin Provincial Department of Education (NO. JJKH20170644sk "Research on the Strategies of Mobile Communication in Jilin Province") for its financial and technical support.

\section{References}

[1]. Su Chun, Li Meiyun. Industry integration of animation industry and mobile communication service industry: Take Japan's mobile game industry as an example [J]. Journal of Guangdong Institute of Public Administration. 2008 (04)

[2]. WEI Qiang, CHEN Sihan. On the integration of Chinese animation industry in colleges and universities_-A case study of college animation community [J]. Journal of Hunan University of Technology (Social Sciences). 2017(05).

[3]. He Yingxi. On the Transformation and Development Strategy of Animation Industry under the Perspective of Anime IP Upgrade__ Taking Shanghai Fine Arts Film Studio as an Example[J]. Movie Review 2017 (18).

[4]. Li Zhonghe, Wang Dan, Xu Wei, Lu Xuedong. Comparison of the development of animation between China and Japan and its enlightenment to China's animation industry[J]. Chinese National Exhibition.2017(10).

[5]. Lin Luxi, Jin Jing, Gao Lijuan, Shen Kaiyuan. Research on the Strategy of Chinese Animation Industry Development Based on New Media Perspective[J]. Strait Science and Technology and Industry. 2016(06) 